\title{
A Derived Relations Analysis of Computer Gaming Complexity
}

\author{
Conor Linehan \\ University of Lincoln, UK \\ Bryan Roche \\ National University of Ireland, Maynooth \\ lan Stewart \\ National University of Ireland, Galway
}

\begin{abstract}
Computer game designers have frequently identified game complexity or "challenge" as a key variable in determining the level of enjoyment users derive from computer games. However, no technical and psychological definition of complexity exists in the gaming literature. The current paper reports on an experiment designed to test a derived relations approach to defining game complexity. Twenty three participants were recruited and exposed to a stimulus equivalence training procedure, designed to train two five-member equivalence relations. Subsequently, participants were presented with a simulated computer game in which they were required to demonstrate responding in accordance with both one-node and three-node derived stimulus equivalence relations under severe time constraints in order to gain a high score. Participants demonstrated consistently correct responding in accordance with derived relational responding. In addition, significantly more correct responses were produced at the relationally less complex (i.e., one-node) level. It is suggested that a derived relations analysis of game complexity may serve as a viable framework within which to investigate the complex processes involved in game playing.
\end{abstract}

Key words: Derived relational responding, computer game, complexity, challenge, enjoyment.

Appropriate 'challenge' and 'complexity' are often proposed as an explanation of why a given computer game succeeds in maintaining player attention and enjoyment across the period of game play (i.e., Davis, Steury, \& Pagaluyan, 2005; Gingold, 2005; Kiili, 2005; Koster, 2005; Malone, 1982; Morlock, Yando, \& Nilogean, 1985; Vorderer, Hartmann, \& Klimmt, 2003). However, there has been little or no scientific psychological research conducted on functionally

Acknowledgements: This work was supported by the Irish Research Council for Science and Engineering Technologies/ Embark Initiative under grant number IRCSET/SC/04/CS0289. The research was conducted by the first author as part of his doctoral research under the supervision of the second author.

Correspondance regarding this article should be addressed to Bryan Roche, Dept of Psychology, NUI Maynooth, Maynooth, Co. Kildare, Ireland. E-mail: bryan.t.roche@nuim.ie defining this concept. We suggest that defining game complexity in terms of derived relational responding allows for a functional-analytic experimental analysis of complexity in game play, and its effect on game user enjoyment. Before we provide the reader with this definition, it is first necessary to provide a brief overview of the concept of derived relations.

One example of derived relational responding is stimulus equivalence (Sidman, 1994; Sidman \& Tailby, 1982;). Sidman reported that training a series of related conditional discriminations using a matching-to-sample (MTS) procedure could result in a number of untrained or derived conditional discriminations systematically related to those that were trained. 
For example, he showed that once participants had been explicitly taught to choose a stimulus " $B$ " in the presence of a stimulus "A," and also to choose a third stimulus " $\mathrm{C}$ " in the presence of that stimulus "B," then a number of untrained responses emerged as follows: Choosing " $\mathrm{A}$ " in the presence of " $A$," " $B$ " in the presence of "B," and "C" in the presence of "C" (referred to by Sidman as reflexivity); choosing " $\mathrm{A}$ " in the presence of " $B$," and " $B$ " in the presence of " $C$ ", (referred to as symmetry); choosing " $\mathrm{C}$ " in the presence of " $A$ " (referred to as transitivity) and "A" in the presence of "C," (combined symmetry and transitivity). Sidman referred to a pattern involving reflexivity, symmetry and transitivity as stimulus equivalence (see also Fields \& Verhave, 1987). In addition, and more importantly, it has been well established that response functions established for one member of an equivalence relation often transfer to other members of the relation without reinforcement (see Dymond \& Rehfeldt, 2000).

The concepts of derived equivalence relations and transfer of stimulus functions allow us to consider how various games may require users to respond to game characters that participate in a wide variety of relations with each other. More specifically, imagine a first person shooter (FPS) game such as Ghost Squad, (Sega', 2007) that requires differential responding to on-screen characters. Points are earned for saving one type of character via one response on the keyboard or other user-interface, and destroying another type of character via a different response. Such a game requires no more complex activities than simple stimulus discrimination.

Now, let us imagine that more characters are introduced as allies of those two original characters. The game requires that the user respond to these new characters not based on their appearance, but on their arbitrary relationship (i.e., derived stimulus equivalence) to the original characters. Let us call the two original characters A1 and A2. Two new characters, labeled B1 and B2, are introduced as 'allies' of $A 1$ and $A 2$, respectively. Now two further characters, $\mathrm{C} 1$ and $\mathrm{C} 2$, are introduced as allies of $\mathrm{B} 1$ and $\mathrm{B} 2$, respectively. These relationships can be established most easily in the game through narrative instructions, as they often are in complex strategy games, or simply through interaction with the game (i.e., trial and error). The important point is that no relation between the $\mathrm{A}$ and $\mathrm{C}$ characters is explicitly specified at any stage. Nevertheless, most game players will have little difficulty responding to the $\mathrm{C}$ characters appropriately (i.e., in the same way they do for the A characters). This, in effect, is an example of the transfer of the response functions of the A stimuli to the $\mathrm{C}$ stimuli via a derived equivalence relation.

The concept of derived relational responding allows us to address the issue of differentiating games in terms of complexity. Specifically, nodal distance may be used to differentiate games that require more or less complex forms of derived relational responding in order to play them successfully. Nodal distance may be best explained as follows. Consider a procedure in which a stimulus $\mathrm{A} 1$ is matched with $\mathrm{B} 1$, then $\mathrm{B} 1$ is matched with $\mathrm{C} 1$, and so on, until the fivemember equivalence relation A1-B1-C1-D1-E1 has been formed. The derived transitive relation between $\mathrm{C} 1$ and $\mathrm{A} 1$ can then be described as a one-node derived relation, because one stimulus separates the A stimulus from the $\mathrm{C}$ stimulus in the linear relation between them. The derived relation between the A stimulus and the E stimulus is a three-node relation, because three stimuli separate A1 and E1.

Crucially, nodal distance has been identified as a key determinant of performance on equivalence tests (Arntzen \& Holth, 1997, 2000; Fields, Adams, Verhave \& Newman, 1990; Fields, Landon-Jimenez, Buffington \& Adams, 1995; Fields, Reeve, Rosen, Varelas, Adams, Belanich, \& Hobbie, 1997;). Participants have greater difficulty deriving equivalence relations involving a larger number of nodes, as measured by both accuracy and response times. Thus, using the concept of nodal distance, it appears possible to create games that are topographically identical but vary in terms of the complexity of derived relational responding required to solve them. For example, consider the FPS game involving stimulus equivalence that was discussed above. In order to generate a game that is functionally more complex than the game described, 
we need only introduce further characters, (i.e., D1, D2, E1, and E2) as allies of A1 and A2 respectively. The crucial point is the manner in which these characters are related. In the example above, the A characters were introduced as allies of the $\mathrm{B}$ characters and the $\mathrm{B}$ characters were introduced as allies of the $\mathrm{C}$ characters. The relationship between the $\mathrm{A}$ and $\mathrm{C}$ characters constitutes a one-node equivalence relation. In a more complex game, the $\mathrm{D}$ characters are introduced as related to the $\mathrm{C}$ characters and the $\mathrm{E}$ characters are related to the $\mathrm{D}$ characters. The relationship between the $\mathrm{A}$ and $\mathrm{E}$ characters constitutes a three-node equivalence relation. In neither game is the relation between the 'A' and any other characters, except the ' $\mathrm{B}$ ' characters, explicitly specified at any stage, and thus, both games require derived relational responding for successful performance. However, the game that requires participants to respond to three-node equivalence relations is functionally more complex than the game that requires participants to respond to one-node relations.

Game complexity represents a variable that is immediately amenable to laboratory-based end-user analysis. Derived relational responding appears to represent a useful framework within which to conduct research on this topic. In order to test this assumption an experiment was conducted, in which participants were first presented with training and testing designed to establish two five-member stimulus equivalence relations using a range of stimuli to be employed in a subsequent computer game. Once completed, participants played a game consisting of levels that varied in terms of the complexity of derived relational responding required to play them (i.e., nodal distance). Specifically, participants played both a one-node and three-node equivalence relation game. Response accuracy was employed as a dependent measure in all phases.

\section{Method}

\section{Participants}

Participants were 23 undergraduate students, 11 female and 12 male. Participants were offered a payment of $€ 5$ upon completion of the game.

\section{Materials}

Stimulus presentation and recording of responses was controlled by Microsoft Visual Basic 6.0 software (see Cabello, Barnes-Holmes, O’Hora, \& Stewart, 2002; Dixon \& MacLin, 2003) presented on a computer screen (resolution 1024 x 768). Two nonsense syllables, "JOM," and, "VEK," and eight colored geometric shapes (a red circle, green square, yellow pentagon, blue triangle, black crescent, white cylinder, cyan cross and orange arrow) were used as stimuli.

\section{Design}

The experiment employed a repeated measures design with complexity as the withinparticipants variable and number of correct responses (i.e., score) on each level of the game as the dependent measure.

\section{Procedure}

The study was divided into two phases; the stimulus equivalence training and testing phase, and the game phase.

\section{Stimulus equivalence training and testing}

Participants first received stimulus equivalence training and testing. Two three-node stimulus relations (A1-B1-C1-D1-E1 and A2B2-C2-D2-E2) were established using a blocked matching-to-sample procedure, in which each conditional discrimination (i.e., two tasks) was trained to criterion individually and in succession. During each trial, the sample and comparison stimuli were presented simultaneously. These stimuli remained on-screen until participants registered a response. On training trials, participants' responses were followed by corrective feedback that remained on screen for two seconds, followed by a one second intertrial interval (ITI). On testing trials, corrective feedback was absent and participants' responses were followed directly by the one second ITI.

Once participants had passed stimulus equivalence training they were presented with stimulus equivalence testing. The latter involved four tasks presented 10 times each in a quasi-random order, with no more than two consecutive presentations of any task. The test tasks probed for both transitive A-E relations (i.e., A1-E1 and A2-E2) 
and combined transitive and symmetrical E-A relations (i.e., E1-A1 and E2-A2). Participants were required to respond correctly on 39 trials in a single block of 40 in order to pass this phase. If they did not meet this criterion, they were returned to the beginning of the stimulus equivalence training and testing sequence (up to a maximum of three times) until they once again passed the training phase and then the testing phase on their first exposure.

\section{Gaming}

The game consisted of three levels; Level 1 was a training level; Level 2 required participants to respond in accordance with one-node stimulus equivalence to score points; Level 3 required participants to respond in accordance with three-node stimulus equivalence to score points. It should be noted that the order of presentation of Levels 2 and 3 was counterbalanced to eliminate any possible order effects.

As illustrated in Figure 1, the game interface involved a control panel at the bottom of the screen, with the current game level displayed in the left hand corner, the participant's score presented in the right hand corner and a button labeled 'DESTROY!' in the centre of the panel. Stimuli were presented on-screen in quasi-randomized positions for a duration of 2 s each. No more than one stimulus ever appeared on-screen at any one time. The stimuli increased in size by $25 \%$ every 0.5 of a second from the onset of the stimulus presentation, in order to simulate the effect of the stimulus approaching the player in three-dimensional space.

Participants had two available response options in each game level; namely, a save response, which involved clicking on a game character with the mouse pointer, and a destroy response, which involved clicking on the button labeled destroy with the mouse pointer. When a mouse click on either a character or the 'DESTROY!' button was recorded, the character displayed was removed from the screen. If the response was correct, the displayed score was increased by 1 . If the response was incorrect, or no response was recorded within 1.5 seconds of the presenta- tion of the character, the score was reduced by 1 (i.e., negative scoring). Regardless of the response made, a $1000 \mathrm{~ms}$ ITI was initiated after each response and before the presentation of the following trial.

\section{Manipulating Relational Complexity}

In Level 1, the training level, the characters presented were the 'A' stimuli from the previous equivalence training stage. In this level, points were earned for saving the $A 1$ stimulus (a red circle) and destroying the A2 stimulus (a green square) within a 2 second response window. Points were lost for destroying the A1 stimulus, saving the A2 stimulus, or not responding within 2 seconds. Participants played this level until a score of 20 points had been achieved. It should be noted that, due to the negative scoring system employed, there was no limit to the number of trials to which a participant might be exposed in attempting to reach a score of +20 during Level 1 . In order to obtain a score of +20 , consistently correct responding was required.

In Level 2, the one-node equivalence level, the characters that were presented in the game space were the ' $\mathrm{C}$ ' stimuli from the stimulus equivalence training and testing phase. Participants were required to demonstrate responding in accordance with the established equivalence relations in the absence of trialby-trial feedback in order to achieve a high score. For example, just as points were gained in Level 1 for saving the A1 stimulus (a red circle), points were gained in Level 2 for saving the $\mathrm{C} 1$ stimulus (a yellow pentagon), which participated in a derived equivalence relation with the red circle. It must be noted that these points were not displayed to participants on a trial-by-trial basis, as was the case in Level 1. Rather, points for both Levels 2 and 3 were displayed to participants after the completion of both levels.

Level 3, the three-node equivalence level, involved the presentation of the ' $E$ ' stimuli from the stimulus equivalence training phase. Upon the completion of the 48 trials, Level 3 came to an end and the participants' score for both Levels 2 and 3 were presented on-screen. 


\section{Results}

Data for the stimulus equivalence training and testing phase are presented below, followed by data for the gaming phase. Table 1 presents the number of attempts that each participant required to pass each stage in stimulus equivalence training and testing. Participants were required to respond correctly on nineteen out of twenty trials in each training block and thirty nine out of forty trials in each testing block in order to pass that stage.

All participants passed the four stimulus

Table 1. Number of exposures required by each participant to pass each stage of stimulus equivalence training and testing. Where an ' $\mathrm{x}$ ' appears under Testing, this indicates that the relevant participant failed to pass the test for combined symmetry and transitivity on their fourth exposure to the training and testing procedure.

\begin{tabular}{|c|c|c|c|c|c|}
\hline Participant & $\begin{array}{l}\text { Train } \\
\text { A-B }\end{array}$ & $\begin{array}{l}\text { Train } \\
\text { B-C }\end{array}$ & $\begin{array}{l}\text { Train } \\
\text { C-D }\end{array}$ & $\begin{array}{l}\text { Train } \\
\text { D-E }\end{array}$ & $\begin{array}{l}\text { Test A-E } \\
\text { and } E-A\end{array}$ \\
\hline 1 & 2 & 2 & 2 & 2 & 2 \\
\hline 2 & 1 & 1 & 1 & 1 & 1 \\
\hline 3 & 1 & 1 & 3 & 1 & 1 \\
\hline 4 & 3 & 1 & 1 & 2 & 1 \\
\hline 5 & 5 & 4 & 4 & 4 & $\mathrm{x}$ \\
\hline 6 & 4 & 3 & 3 & 3 & 3 \\
\hline 7 & 2 & 3 & 2 & 4 & 2 \\
\hline 8 & 4 & 2 & 3 & 2 & 2 \\
\hline 9 & 1 & 1 & 1 & 1 & 1 \\
\hline 10 & 3 & 2 & 2 & 3 & 2 \\
\hline 11 & 2 & 2 & 2 & 3 & 2 \\
\hline 12 & 1 & 1 & 1 & 1 & 1 \\
\hline 13 & 1 & 1 & 2 & 1 & 1 \\
\hline 14 & 2 & 1 & 1 & 1 & 1 \\
\hline 15 & 2 & 2 & 2 & 2 & 2 \\
\hline 16 & 1 & 1 & 1 & 1 & 1 \\
\hline 17 & 1 & 1 & 2 & 1 & 1 \\
\hline 18 & 2 & 2 & 2 & 4 & 2 \\
\hline 19 & 2 & 2 & 2 & 2 & 2 \\
\hline 20 & 1 & 1 & 1 & 1 & 1 \\
\hline 21 & 3 & 2 & 4 & 2 & 2 \\
\hline 22 & 2 & 1 & 1 & 1 & 1 \\
\hline 23 & 3 & 2 & 2 & 2 & 2 \\
\hline
\end{tabular}

equivalence training blocks and proceeded to the stimulus equivalence test. Eleven participants passed the stimulus equivalence test on their first exposure, a further ten passed on their second exposure, one passed on his third exposure and only one participant (P5) failed to pass within four exposures. This participant was not exposed to the subsequent phase of the experiment.
Twenty two participants advanced to the gaming phase. The gaming phase consisted of three separate levels; the training level, the one-node equivalence level and the three-node equivalence level. In the training level, participants were required to attain a score of 20 points in order to proceed to the subsequent game levels. Participants who made a large number of incorrect responses during this level were exposed to more trials than participants who produced predominantly correct responses. As a result, the number of trials presented to each participant in the training level varied considerably, as is illustrated in Table 2 .

Table 2. Number of trials required by each participant to reach the criterion for passing the training level.

\begin{tabular}{rrrrrrrrrrr}
\hline P1 & P2 & P3 & P4 & P6 & P7 & P8 & P9 & P10 & P11 & P12 \\
\hline 24 & 36 & 22 & 32 & 26 & 24 & 30 & 22 & 36 & 24 & 60 \\
P13 & P14 & P15 & P16 & P17 & P18 & P19 & P20 & P21 & P22 & P23 \\
20 & 24 & 22 & 22 & 24 & 27 & $>80$ & $>80$ & 32 & 37 & 24 \\
\hline
\end{tabular}

While most participants passed the training level within thirty trials, there were a few exceptions. For example, Participant 12 required sixty trials to reach the criterion, while both Participants 19 and 20 required more than eighty trials to reach criterion. Data obtained from Participants 19 and 20 were considered outliers and will not be included in the following analysis.

Table 3 presents the number of correct responses made by each participant in the onenode level. A score of 43 represented a $90 \%$ correct response criterion. Seventeen of the twenty participants passed the one-node game at the $90 \%$ correct criterion. Thus, it appears that the majority of participants consistently demonstrated equivalence responding in this game level. Of the three participants who did not reach the $90 \%$ criterion, none failed to respond within the time limit on any trial. However, it appears that they were responding to the stimuli on some basis other than that of previous equivalence training.

Table 4 presents the number of correct responses made by each participant in the three-node level. As in the case of the one-node 
Table 3. Number of trials in which correct, incorrect and no responses were recorded in the one node level.

\begin{tabular}{|c|c|c|c|}
\hline $\begin{array}{c}\text { Participant } \\
\text { Number }\end{array}$ & Correct & Incorrect & $\begin{array}{c}\text { No } \\
\text { Response }\end{array}$ \\
\hline 1 & 48 & 0 & 0 \\
\hline 2 & 3 & 45 & 0 \\
\hline 3 & 44 & 4 & 0 \\
\hline 4 & 1 & 47 & 0 \\
\hline 6 & 48 & 0 & 0 \\
\hline 7 & 47 & 1 & 0 \\
\hline 8 & 45 & 0 & 3 \\
\hline 9 & 47 & 1 & 0 \\
\hline 10 & 47 & 1 & 0 \\
\hline 11 & 47 & 0 & 1 \\
\hline 12 & 4 & 44 & 0 \\
\hline 13 & 47 & 0 & 1 \\
\hline 14 & 48 & 0 & 0 \\
\hline 15 & 48 & 0 & 0 \\
\hline 16 & 47 & 0 & 1 \\
\hline 17 & 48 & 0 & 0 \\
\hline 18 & 48 & 0 & 0 \\
\hline 21 & 48 & 0 & 0 \\
\hline 22 & 48 & 0 & 0 \\
\hline 23 & 48 & 0 & 0 \\
\hline
\end{tabular}

Table 4. Number of trials in which correct, incorrect and no responses were recorded in the three node level.

\begin{tabular}{cccc}
\hline $\begin{array}{c}\text { Participant } \\
\text { Number }\end{array}$ & Correct & Incorrect & $\begin{array}{c}\text { No } \\
\text { Response }\end{array}$ \\
1 & 0 & 48 & 0 \\
2 & 1 & 47 & 0 \\
3 & 46 & 2 & 0 \\
4 & 1 & 47 & 0 \\
6 & 47 & 1 & 0 \\
7 & 1 & 47 & 0 \\
8 & 48 & 0 & 0 \\
9 & 48 & 0 & 0 \\
10 & 48 & 0 & 0 \\
11 & 47 & 0 & 1 \\
12 & 4 & 44 & 0 \\
13 & 47 & 0 & 1 \\
14 & 0 & 48 & 0 \\
15 & 0 & 48 & 0 \\
16 & 48 & 0 & 0 \\
17 & 48 & 0 & 0 \\
18 & 47 & 0 & 1 \\
21 & 48 & 0 & 0 \\
22 & 47 & 0 & 1 \\
23 & 48 & 0 & 0 \\
\hline
\end{tabular}

game, a score of 43 or above represented a $90 \%$ correct response criterion. Thirteen participants passed the three-node level at the $90 \%$ correct response criterion. It appears that the majority of participants consistently demonstrated equivalence responding in this game level. Similarly to Level 2, all participants who failed to reach the 90\% criterion actually responded incorrectly on $90 \%$ of trials. These participants did not simply fail to respond to the stimuli presented but produced reversed transfer of functions.

Responses were recorded on 1,910 out of a total of 1,920 trials presented to all participants across Levels 2 and 3. Only $0.5 \%$ of trials presented failed to evoke a response. Thus, it would appear that the relatively short and simple game presented in the current study was sufficient in complexity to maintain participants' engagement with the game.

Participants appeared less likely to produce response patterns consistent with stimulus equivalence training during the three-node derived relational level of the game than during the one-node level. In order to test whether the difference in correct responding observed across the one-node $(\mathrm{M}=40.55, \mathrm{SD}=16.37)$ and three-node $(M=31.2 S D=22.8)$ game levels was statistically significant, a repeated measures ANOVA was carried out to compare these data. There was a significant difference between the number of correct responses recorded for participants across the one-node and three-node games (Wilks' Lambda $=0.807$, $F(1,19)=4.549, p=0.046)$. Furthermore, the magnitude of differences in means was large (eta squared $=0.193$ ).

In the current study the majority of participants displayed derived relational responding in accordance with both one-node and threenode equivalence in the context of a heavily time constrained game playing environment (i.e., using unconventional stimuli, stimulus presentation formats, and temporal constraints on responding). In addition, participants produced significantly more correct responses during the game based on a less complex derived relational responding task (i.e., one-node), than during the game based on a more complex task (i.e., three-node). 


\section{Discussion}

The current experiment demonstrates how important features of computer game playing may be understood and analyzed in behavioral terms. Specifically, the current study examined the concept of game 'challenge' or complexity, which has been previously identified as a key determinant in the appeal of individual games (i.e., Davis, Steury, \& Pagaluyan, 2005; Gingold, 2005; Kiili, 2005; Malone, 1982; Morlock, Yando, \& Nilogean, 1985; Vorderer, Hartmann, \& Klimmt, 2003). A novel definition of game 'challenge,' or complexity was examined in the current study. It was proposed that games that require participants to derive relations between game stimuli that are further apart in terms of nodal distance are more difficult and challenging than games that require participants to derive relations that are closer together in terms of nodal distance. The current study found that relational complexity significantly affected the correct responses recorded by participants while playing the game. Specifically, participants attained lower scores in the game level that required three-node derived equivalence responding than in the one-node level.

The finding that participants readily demonstrated derived relational responses within a time constrained game playing environment improves the ecological validity of a derived relations paradigm for the analysis of games, because real-world games typically involve time constraints and yet require high fluency levels in responding in order to master the game. Indeed, the current study suggests that derived relational responding alone may represent an engaging task to complete within a computer gaming context. Specifically, participants recorded a response, regardless of whether it was correct or incorrect, on 1910 out of a total of 1,920 trials presented to all participants across levels 2 and 3 of the current study. Only $0.5 \%$ of trials presented failed to evoke a response. Thus, it would appear that the relatively short and simple game presented in the current study was sufficient in complexity to maintain participants' engagement. Of course, modern computer games typically involve such relationally complex stimulus features wrapped in impressive presentation formats such as realistic graphic rendering. Additionally, it must be noted that participants were paid $€ 5$ for completion of the study and this cannot be ruled out as a contributing factor in participants' observed engagement with the game. Nevertheless, at a psychological level we may now perhaps begin to conceive of computer game play as involving the features of stimulus control and the derived transfer of discriminative control, amongst other features.

It might be noted that effects of relational complexity may have been confounded with the delivery of a stimulus equivalence test prior to game play. Specifically, during the stimulus equivalence testing phase, probes for the threenode $\mathrm{A}-\mathrm{E}$ and $\mathrm{E}-\mathrm{A}$ relations were presented, while probes for the one-node A-C and C-A relations were not. Thus, participants had previous experience of deriving the three-node relations before playing the game. It may be the case, therefore, that game play proficiency was somewhat enhanced during the three-node game. Despite this, however, participants produced significantly more correct responses during the one-node game. Thus, it is possible that there would have been an even greater difference between the one-node and threenode game scores if the stimulus equivalence test had not been delivered.

One interesting issue with regard to the observed response patterns is that when a non-predicted response pattern was observed, it was typically nearly $100 \%$ incorrect across a test phase. More specifically, across both levels 2 and 3 of the game, all participants produced responses that were either $>90 \%$ correct or $>90 \%$ incorrect. In effect, it appears that subjects confused the two equivalence relations. It would appear, therefore, that for no subject were the equivalence relations irrelevant during game play, even where poor control by derived relations was observed. It is important to understand, however, that precisely such weakness in control over game responses by derived relations was predicted in attempting to generate a challenging computer game (i.e., if all responses were to be perfectly fluent and perfectly controlled by derived 
relations the game would not be challenging at all). The important issue, therefore, is the relative difference in strength of control by the one-node and three-node derived relations across the game phases. This appears to have been clearly demonstrated.

An interesting conclusion from the current work is that the methods of behavior analysis may represent a useful framework through which to analyze computer game playing. While traditional direct contingency explanations of behavior alone may improve our understanding of game playing behavior to some extent, (see Loftus \& Loftus, 1983; Siang \& Rao, 2003) the current study demonstrates that complex derived relational processes must also be examined in any rigorous behavioral analysis of modern computer game playing. Indeed, a viable and potentially fruitful definition of game challenge in terms of relational complexity can now be offered to those researching game complexity in both academic and industrial settings. Specifically, experimental analyses may now be conducted using nodal distance as an operational definition of relational complexity. The application of further and more complex types of derived relational responding, such as relations of opposition, difference and comparison (see Hayes, Barnes-Holmes, \& Roche, 2001), to the analysis of computer game play, may help to further our understanding of the role of various other types of relational responding in gaming. The current findings represent a solid first step to understanding functionally the relationship between derived relational processes and game performance and experience. In addition, behavior analysis may provide an ideal framework within which to develop novel, objective behavioral and physiological measures of enjoyment that may prove suitable for the analysis of computer games and computer game playing.

\section{References}

Arntzen, E, \& Holth, P. (1997) Probability of stimulus equivalence as a function of training design. The Psychological Record, 47, 309-320.

Arntzen, E., \& Holth, P. (2000). Equivalence outcome in single subjects as a function of training structure. The Psychological Record, 50, 603-628.

Bauckhage, C., Thurau, C., \& Sagerer, G. (2003). Learning human-like opponent behavior for interactive computer games. In B. Michaelis, \& G. Krell (Eds.) Pattern Recognition, Lecture Notes in Computer Science, pp. 148-155. Springer: Berlin / Heidelberg. Cabello, F., Barnes-Holmes, D., O’Hora, D., \& Stewart, I. (2002). Using visual basic in the experimental analysis of human behavior: A brief introduction. Experimental Analysis of Human Behavior Bulletin, 20, 17-20

Davis, J.P., Steury, K., \& Pagulayan, R. (2005). A survey method for assessing perceptions of a game: The consumer playtest in game design. The International Journal of Computer Game Research, 5, Retrieved May 1, 2009 from http://gamestudies.org/0501/ davis_steury_pagulayan/

Dixon, M.R., \& MacLin, O.H. (2003). Visual Basic for Behavioural Psychologists. Reno, NV: Context Press.

Dymond, S., \& Rehfeldt, R.A. (2000). Understanding complex behavior: The transformation of stimulus functions. The Behavior Analyst, 23, 239-254.

Fields, L., Adams, B.J., Verhave, T., \& Newman, S. (1990). The effects of nodality on the formation of equivalence classes. Journal of the Experimental Analysis Of Behavior 53, 345-358.

Fields, L., Landon-Jimenez, D. V., Buffington, D. M., \& Adams, B. J. (1995) Maintained nodal-distance effects in equivalence classes. Journal of the Experimental Analysis of Behavior, 64, 129-145.

Fields, L., Reeve, K. F., Rosen, D., Varelas, A., Adams, B.J., Belanich, J., \& Hobbie, S.A. (1997). Using the simultaneous protocol to study equivalence class formation: The facilitating effects of nodal number and size of previously established equivalence classes. Journal of the Experimental Analysis of Behavior, 67, 367-389.

Fields, L., \&Verhave, T. (1987). The structure of equivalence classes. Journal of the Experimental Analysis of Behavior, 48, 317-332. 
Gingold, C. (2005). What Warioware can teach us about game design. International Journal of Computer Games Research, 5, October 2005. Retrieved May 1, 2009 from http:// gamestudies.org/0501/gingold/

Hayes, S.C., Barnes-Holmes, D., \& Roche, B. (Eds.) (2001). Relational Frame Theory: A Post-Skinnerian Account of Human Language and Cognition. New York: Kluwer Academic/Plenum Publishers.

Jørgensen, K. (2003). Problem solving: THe essence of player action in computer games. In Copier, Marinka \& Joost Raessens (Eds.): Proceedings from Level Up: Digital Games Research Conference 2003. Utrecht: Universiteit Utrecht, The Netherlands. Retrieved May 1, 2009 from http://www. digra.org/dl/db/05150.49599.pdf

Kiili, K. (2005). Digital game-based learning: Towards an experiential gaming model. The Internet and Higher Education, 8, 13-24.

Koster, R. (2005). A theory of fun for game design. Scottsdale, AZ: Paraglyph Press.

Lindsley, O. (1992). Precision teaching: discoveries and effects. Journal of Applied Behavior Analysis, 25, 51-57.

Loftus, G.R.,\& Loftus, E.F. (1983). Mind at play: The Psychology of Video Games. New York: Basic Books.

Malone, T. W. (1982). Heuristics for designing enjoyable user interfaces: lessons from computer games. In Proceedings of the 1982 conference on Human factors in computing systems, (pp. 63-68). New York, NY, USA: ACM

Morlock, H., Yando, T., \& Nigolean, K., (1985). Motivation of video game players, Psychological reports, 57, 247-250.
Sega (Developer). (2007). Ghost Squad: Wii Edition. Japan: Sega.

Siang, A.C., \& Rao, R.K. (2003). Theories of learning: A computer game perspective. Proceedings of the IEEE Fifth International Symposium on Multimedia Software Engineering, pp. 239-245. Taipei, Taiwan.

Skinner, B.F. (1938). The Behavior of Organisms. New York: Appleton-Century-Crofts. Skinner, B.F. (1968). The Technology of Teaching. New York: Appleton-Century-Crofts.

Skinner, B. F. (1969). Contingencies of reinforcement: A theoretical analysis. New York: Appleton-Century-Crofts.

Sidman, M. (1971). Reading and auditoryvisual equivalences. Journal of Speech and Hearing Research, 14, 5-13.

Sidman, M. (1994). Equivalence Relations and Behavior: A Research Story. Boston: Authors Cooperative.

Sidman, M., Rauzin, R., Lazar, R., Cunningham, S., Tailby, W., \& Carrigan, P. (1982). A search for symmetry in the conditional discriminations of rhesus monkeys, baboons, and children. Journal of the Experimental Analysis of Behavior, 37, 23-44.

Sidman, M., \& Tailby, W. (1982). Conditional discrimination versus matching to sample: An extension of the testing paradigm. Journal of the Experimental Analysis of Behaviour, 37, 5-22.

Vorderer, P., Hartmann, T., \& Klimmt, C. (2003). Explaining the enjoyment of playing video games: The role of competition. In ICEC '03: Proceedings of the second international conference on Entertainment computing, (pp. 1-9). Pittsburgh, PA, USA: Carnegie Mellon University. 